\title{
APPLICATIONS OF A NEW MODEL FOR THE DIFFERENTIAL CROSS-SECTION OF A CLASSICAL POLYATOMIC GAS
}

\author{
Carlo Cercignani and Maria Lampis \\ Dipartimento di Matematica \\ Politecnico di Milano, I-20124 Milano, Italy \\ Jens Struckmeier \\ Fachbereich Mathematik \\ Universität Kaiserslautern, D-67653 Kaiserslautern, Germany
}

\begin{abstract}
We give a comparison of various differential cross-section models for a classical polyatomic gas for a homogeneous relaxation problem and the shock wave profiles at Mach numbers 1.71 and 12.9. Besides the standard Borgnakke-Larsen model and its generalization to an energy dependent coefficient to control the amount of rotationally elastic and completely inelastic collision, we discuss some new models recently proposed by the same authors. Moreover, we present numerical algorithms to implement the models in a particle or Monte-Carlo code and compare the numerical shock wave profiles with existing experimental data.
\end{abstract}

\section{Introduction}

The Boltzmann collision operator for a polyatomic rarefied gas, where each molecule carries one additional continuous variable describing the rotational energy of the molecule may be written in the form

$$
Q(f)=\iint_{\mathbb{R}^{3}} \int_{S^{2}} \int_{\mathcal{E}+\mathcal{E}_{1} \leq E}\left|\mathbf{v}-\mathbf{v}_{1}\right| \sigma\left(E ; \mathbf{e} \cdot \mathbf{e}^{\prime} ; \mathcal{E}, \mathcal{E}_{1} \rightarrow \mathcal{E}^{\prime}, \mathcal{E}_{1}^{\prime}\right)\left(f^{\prime} f_{1}^{\prime}-f f_{1}\right) \mathrm{d} \mathcal{E}_{1} \mathrm{~d} \mathcal{E} \mathrm{d} \mathbf{e d} \mathbf{v}_{1}
$$

where $f=f(t, \mathbf{x}, \mathbf{v}, \mathcal{E})$ etc. and $\sigma\left(E, \mathbf{e} \cdot \mathbf{e}^{\prime} ; \mathcal{E}, \mathcal{E}_{1} \rightarrow \mathcal{E}^{\prime}, \mathcal{E}_{1}^{\prime}\right)$ denotes the differential cross-section. One further assumes, that the differential cross-section is expressed as the product of the total cross-section $\sigma_{\text {tot }}$, which only depends on the total collision energy $E=\left|\mathbf{v}-\mathbf{v}_{1}\right|^{2} / 4+\mathcal{E}+\mathcal{E}_{1}$, times a transition probability density $\theta\left(\varepsilon, \varepsilon_{1} \rightarrow \varepsilon^{\prime}, \varepsilon_{1}^{\prime}\right)$ depending only on the scaled rotational energies $\varepsilon, \varepsilon_{1}$ and $\varepsilon^{\prime}, \varepsilon_{1}^{\prime}$, before and after the 
collisions $(\varepsilon=\mathcal{E} / E$ etc. $)$, such that the differential cross-section may be expressed as

$$
\sigma\left(E ; \mathbf{e} \cdot \mathbf{e}^{\prime} ; \mathcal{E}, \mathcal{E}_{1} \rightarrow \mathcal{E}^{\prime}, \mathcal{E}_{1}^{\prime}\right)=\frac{\sigma_{t o t}(E)}{4 \pi E^{2 \mu+2}} \theta\left(\varepsilon, \varepsilon_{1} \rightarrow \varepsilon^{\prime}, \varepsilon_{1}^{\prime}\right)
$$

Here we already assume isentropic scattering with respect to the angular dependence in a collision and we will restrict in the following to this model. A more general form for (1.1) is given by

$$
\sigma\left(E ; \mathbf{e} \cdot \mathbf{e}^{\prime} ; \mathcal{E}, \mathcal{E}_{1} \rightarrow \mathcal{E}^{\prime}, \mathcal{E}_{1}^{\prime}\right)=\frac{\sigma_{t o t}(E)}{E^{2 \mu+2}} \varphi\left(\mathbf{e} \cdot \mathbf{e}^{\prime}\right) \theta\left(\varepsilon, \varepsilon_{1} \rightarrow \varepsilon^{\prime}, \varepsilon_{1}^{\prime}\right)
$$

with $\mathbf{e}=\left(\mathbf{v}-\mathbf{v}_{1}\right) /\left|\mathbf{v}-\mathbf{v}_{1}\right|$ and $\mathbf{e}^{\prime}=\left(\mathbf{v}^{\prime}-\mathbf{v}_{1}^{\prime}\right) /\left|\mathbf{v}^{\prime}-\mathbf{v}_{1}^{\prime}\right|$, where the function $\varphi$ describes the angular scattering, e.g.,

$$
\varphi\left(\mathbf{e} \cdot \mathbf{e}^{\prime}\right)=\frac{\alpha}{4 \pi}\left(\frac{1+\mathbf{e} \cdot \mathbf{e}^{\prime}}{2}\right)^{\alpha-1}, \quad \alpha>0
$$

which yields a model different from isentropic scattering.

The main task in the modelling of polyatomic gases is to define an appropriate transition probability density $\theta\left(\varepsilon, \varepsilon_{1} \rightarrow \varepsilon^{\prime}, \varepsilon_{1}^{\prime}\right)$, which should be simple enough to be handled by numerical simulation codes. The basic assumptions on the density $\theta\left(\varepsilon, \varepsilon_{1} \rightarrow \varepsilon^{\prime}, \varepsilon_{1}^{\prime}\right)$ are as follows

1) The function $\theta\left(\varepsilon, \varepsilon_{1} \rightarrow \varepsilon^{\prime}, \varepsilon_{1}^{\prime}\right)$ is positive for all admissible values of the (scaled) rotational energies

2) the function $\theta\left(\varepsilon, \varepsilon_{1} \rightarrow \varepsilon^{\prime}, \varepsilon_{1}^{\prime}\right)$ fulfills the normalization conditions

$$
\int_{0}^{1} \int_{0}^{1-\varepsilon^{\prime}} \theta\left(\varepsilon, \varepsilon_{1} \rightarrow \varepsilon^{\prime}, \varepsilon_{1}^{\prime}\right) \mathrm{d} \varepsilon_{1}^{\prime} \mathrm{d} \varepsilon^{\prime}=1
$$

for all pre-collisional (scaled) rotational energies with $\varepsilon+\varepsilon_{1} \leq 1$

3) the detailed balance relation holds, i.e.

$$
\left(1-\varepsilon-\varepsilon_{1}\right) \theta\left(\varepsilon, \varepsilon_{1} \rightarrow \varepsilon^{\prime}, \varepsilon_{1}^{\prime}\right)=\left(1-\varepsilon^{\prime}-\varepsilon_{1}^{\prime}\right) \theta\left(\varepsilon^{\prime}, \varepsilon_{1}^{\prime} \rightarrow \varepsilon, \varepsilon_{1}\right)
$$

In the following section we will discuss some suitable models for the transition probability density and compare the various models in a homogeneous realaxation problem as well as in one-dimensional shock wave simulations in polyatomic gases. 


\section{Cross-Section Models for Polyatomic Gases}

In the first part of this section we discuss the most widely used cross-section model for polyatomic gases, namely the Borgnakke-Larsen model. Moreover, we generalize the original model to an energy-dependent coefficient, which is necessary to automatically adapt the model to the temperature dependent relaxation times of rotational degrees of freedom. In Section 2.2 the so-called Cercignani-Lampis-type models, which were recently introduced by the same authors in order to obtain a better fit of the resulting transport coefficient to experimental data. For both models we give numerical algorithms how to implement the translational and rotational energy exchange in a numerical simulation code for rarefied gas flows.

\subsection{The Borgnakke-Larsen-Type Models}

The Borgnakke-Larsen model (and its extensions) is the most popular model used in nearly all numerical simulations for polyatomic rarefied gases. It is a phenomenological model to describe the energy exchange between translational and internal degrees of freedom during binary collisions among the gas. In its original form, the Borgnakke-Larsen model considers one continuous variable describing the rotational energy of a molecule; later on, the model was generalized to one discrete rotational energy [8] as well as to vibrational states [2], [10] and chemical reactions (e.g., [14]). The main idea of the Borgnakke-Larsen model - here we restrict ourselve to the case of one continuous variable describing the rotational energy - is to include the different relaxation times of translational and rotational degrees of freedom by assuming that the differential cross-section can be expressed as a linear combination of (rotational) elastic and inelastic collisions, i.e.

$$
\theta_{B L}(\cdot)=(1-a) \theta_{e l}(\cdot)+a \theta_{i n}(\cdot)
$$

where $\theta_{e l}$ and $\theta_{i n}$ denote the elastic and inelastic cross-sections, respectively,

$$
\begin{aligned}
& \theta_{e l}\left(\varepsilon^{\prime}, \varepsilon_{1}^{\prime}, \varepsilon, \varepsilon_{1}\right)=\varepsilon^{-\mu} \varepsilon_{1}^{-\mu} \delta\left(\varepsilon-\varepsilon^{\prime}\right) \delta\left(\varepsilon_{1}-\varepsilon_{1}^{\prime}\right) \\
& \theta_{i n}\left(\varepsilon^{\prime}, \varepsilon_{1}^{\prime}, \varepsilon, \varepsilon_{1}\right)=\frac{(2 \mu+3) !}{(\mu !)^{2}}\left(1-\varepsilon-\varepsilon_{1}\right),
\end{aligned}
$$

The parameter $a$ appearing in (2.1) is related with the rotational relaxation collision number $Z_{r a t}[2]$ via the relation $a=1 / Z_{\text {rat }}$ and in the original form of the Borgnakke-Larsen model, the parameter $a$ is assumed to be constant during a numerical simulation (typically, one uses $a=0.2$ for nitrogen, as proposed by Bird $[2])$.

On the other hand, it is well-known, that the rotational relaxation collision number $Z_{\text {rat }}$ is a temperature-dependent quantity and an appropriate model for the temperature dependence was given by Parker [12]. Hence, to reproduce the correct relaxation times of rotational degrees of freedom, it is necessary to include an 
energy-dependent parameter $a=a(E)$ in the Borgnakke-Larsen cross-section defined in (2.1), such that the transition probability density $\theta$ even depends on the total collision energy $E$.

Several authors discussed generalizations of the original BL model to include this energy-dependence: in Ref. [4], Boyd proposed an extended Borgnakke-Larsen model, where the inelastic collision parameter $a$ is temperature-dependent, i.e. $a=a(T)$, where $T$ denotes the temperature of the gas; but the use of a macroscopic quantity in a single microscopic collision seems to be questionable. A more sophisticated model for an energy-dependent parameter $a(E)$ was given by Koura [8] for a discrete internal energy and in Ref. [9] for the case of a continuous internal energy.

In the present paper we will follow the model proposed by Sack in Ref. [14]. Here, the author relates the temperature dependence of the rotational relaxation collision number, defined in the Parker model, with an energy dependent parameter $a=a(E)$ in the Borgnakke-Larsen model. This relation is given via the Laplace transformation, which means that the total collision energy of a single collision and the temperature of the gas are conjugated variables; in the sense, that a temperature dependence of macroscopic quantities yields an energy dependence of the corresponding microscopic ones. This model is referred in the following as the socalled generalized Borgnakke-Larsen model (GBL).

The concrete expression for the energy-dependence of the inelastic collision parameter $a(E)$ depends on the total cross-section as well as on the underlying macroscopic model for the temperature-dependence of the rotational relaxation collision number $Z_{\text {rat }}$, given, e.g., by the Parker model. Moreover, one may relate this number $Z_{\text {rat }}$ to the rotational part of the volume viscosity $\eta_{\text {rot }}$ of the polyatomic gas, which is itself temperature-dependent. We refer the reader to Ref. [14], where one may find explicit expression for the parameter $a(E)$ and varies total cross-section models, like the hard-sphere, the inverse power and the Sutherland potential.

The implementation of the generalized Borgnakke-Larsen model in a numerical simulation code for polyatomic gases is straightforward and may be summarized as follows:

1) for each collision pair, one decides via the total cross-section, whether a collision will occur or not,

2) if two particles will collide, one evaluates the expression $a(E)$ to decide whether the collision is elastic or inelastic, i.e. if $r \leq a(E)$, where $r$ denotes a uniformly distributed random number in $[0,1]$, the collision will be inelastic - otherwise elastic,

3) if the collision is an inelastic one, the post-collisional rotational energies of the 
two particles are given by the formula

$$
\mathcal{E}^{\prime}=\left(1-r_{1}^{1 / 3}\right) E, \quad \mathcal{E}_{1}^{\prime}=\left(1-\sqrt{r_{2}}\right)(E-\mathcal{E})
$$

where $r_{1}$ and $r_{2}$ denote two uniformly distributed random numbers on [0,1]. Then, the reduced total energy $E^{\prime}=E-\mathcal{E}^{\prime}-\mathcal{E}_{1}^{\prime}$ is distributed on the translational degrees of freedom of the two molecules using a standard collision step for a monoatomic gas,

4) if the collision is an elastic one, one performs a standard collision step like for a monoatomic gas, where the rotational energies of the two molecules remain fixed.

\subsection{The Cercignani-Lampis-Type Models}

In Refs. [6], [7], the authors discussed some new models for the transition probability density $\theta\left(\varepsilon, \varepsilon_{1} \rightarrow \varepsilon^{\prime}, \varepsilon_{1}^{\prime}\right)$, which are derived from a quite general construction principle: one starts from a sensible approximate positive kernel $\theta_{0}\left(\varepsilon^{\prime}, \varepsilon_{1}^{\prime}, \varepsilon, \varepsilon_{1}\right)$, chosen on the basis of intuition, which, however, does not satisfy the basic properties given in the introduction. Thus, this approximate kernel is modified by adding some correction terms, which finally yields the following expression for $\theta(\ldots)$,

$$
\left\{\begin{aligned}
\theta(\ldots) & =H\left(\varepsilon^{\prime}, \varepsilon_{1}^{\prime}\right) \theta_{1}(\ldots)+\left(1-H\left(\varepsilon^{\prime}, \varepsilon_{1}^{\prime}\right)\right) \theta_{2}(\ldots) \\
\theta_{1}(\ldots) & =\theta_{0}(\ldots) / H\left(\varepsilon^{\prime}, \varepsilon_{1}^{\prime}\right) \\
\theta_{2}(\ldots) & =\frac{(2 \mu+3) !}{(\mu !)^{2}}\left(1-\varepsilon-\varepsilon_{1}\right)\left(1-H\left(\varepsilon, \varepsilon_{1}\right)\right) / I .
\end{aligned}\right.
$$

where

$$
H\left(\varepsilon^{\prime}, \varepsilon_{1}^{\prime}\right)=\int_{0}^{1} \int_{0}^{1-\varepsilon} \theta_{0}\left(\varepsilon^{\prime}, \varepsilon_{1}^{\prime}, \varepsilon, \varepsilon_{1}\right) \varepsilon^{\mu} \varepsilon_{1}^{\mu} d \varepsilon d \varepsilon_{1}
$$

The expression $H\left(\varepsilon^{\prime}, \varepsilon_{1}^{\prime}\right)$ may even be interpreted as an "accomodation coefficient", like in the Cercignani-Lampis model for the gas-surface interaction [5], where the second term on the right hand side of (2.2) need to be added, because the first term does not satisfy all the fundamental properties of normalization and reciprocity. In Ref. [7] the following expression for the approximate kernel was introduced

$$
\left\{\begin{array}{l}
\theta_{0}\left(\varepsilon^{\prime}, \varepsilon_{1}^{\prime}, \varepsilon, \varepsilon_{1} ; a, b\right)=\frac{2 b a}{\pi} \varepsilon^{-\frac{\mu}{2}} \varepsilon_{1}^{-\frac{\mu}{2}} \varepsilon^{\prime-\frac{\mu}{2}} \varepsilon_{1}^{\prime-\frac{\mu}{2}} \times \\
\times \frac{\left(1-\varepsilon-\varepsilon_{1}\right)}{\left(1-\varepsilon-\varepsilon_{1}\right)+\left(1-\varepsilon^{\prime}-\varepsilon_{1}^{\prime}\right)} \times \exp \left[-a\left(\varepsilon-\varepsilon^{\prime}\right)^{2}-a\left(\varepsilon_{1}-\varepsilon_{1}^{\prime}\right)^{2}\right] .
\end{array}\right.
$$

and the corresponding expressions for the transport coefficients of linear molecules $(\mu=0)$ are given there.

According to this kernel the collisions are inelastic, but not in general maximally 
inelastic. Moreover, in the limit $a \rightarrow \infty$ one has $\theta_{0}(\ldots) \rightarrow \theta_{e l}$, such that the original Borgnakke-Larsen model is included in this model as asymptotic limit. Finally, in the framework of the probability density given by (2.2) one may take into account elastic collisions as well: one may propose another possible kernel as follows:

$$
\left\{\begin{array}{l}
\theta\left(\varepsilon^{\prime}, \varepsilon_{1}^{\prime}, \varepsilon, \varepsilon_{1} ; a, b\right)=\theta_{0}\left(\varepsilon^{\prime}, \varepsilon_{1}^{\prime}, \varepsilon, \varepsilon_{1} ; a, b\right)+ \\
\left(1-H\left(\varepsilon^{\prime}, \varepsilon_{1}^{\prime}\right)\right) \delta\left(\varepsilon-\varepsilon^{\prime}\right) \delta\left(\varepsilon_{1}-\varepsilon_{1}^{\prime}\right) .
\end{array}\right.
$$

Due to the second term, this kernel describes also elastic collisions.

The implementation of the new scattering models in a numerical simulation code for polyatomic gases is quite more complicated then for the generalized BorgnakkeLarsen model. First of all, there exists no analytic expression for the "accomodation coefficient" $H\left(\varepsilon, \varepsilon_{1}\right)$, such that the term has to be evaluated at some discrete values $\left(\varepsilon^{i}, \varepsilon_{1}^{j}\right)$ by numerical integration in order to generate a table of discrete values on the triangle $0 \leq \varepsilon \leq 1,0 \leq \varepsilon_{1} \leq 1-\varepsilon$. Hence, even the inversion method to generate the post-collisional rotational energies, which was applied in the Borgnakke-Larsen model, fails and one has to use some different techniques to generate the postcollisional quantities. Here, we propose to use the so-called acceptance-rejection method, which is shortly explained - together with the inversion method - in the following: suppose that a probability density $f(x)$ is given on $\mathbb{R}_{+}$, such that

$$
\int_{\mathbb{R}} f(x) \mathrm{d} x=1
$$

Then, the distribution function $F(x)$ is given by the integral

$$
F(x)=\int_{0}^{x} f(y) \mathrm{d} y
$$

If one is able to obtain an analytic expression for the distribution function $F(x)$ for all $x \in \mathbb{R}_{+}$, then a uniformly distributed point in the unit interval $[0,1]$ may be transformed into a $f$-distributed one on $\mathbb{R}_{+}$by the inversion formula

$$
x=\min _{y \in \mathbb{R}_{+}}\{y: F(y) \geq r\}
$$

If the inversion method fails, because there exists no analytic expression for the distribution function $F(x)$, one may apply the so-called acceptance-rejection method: suppose that the probability density $g(x)$ on $\mathbb{R}_{+}$is an "easy" density, i.e. the inversion method may be applied to compute $g$-distributed points, and there exists a constant $c \geq 1$, such that

$$
f(x) \leq c g(x), \quad \forall x \in \mathbb{R}_{+}
$$


Then one generates first a $g$-distributed point via the inversion method and accepts this point as $f$-distributed, if the condition

$$
\frac{f(x)}{c g(x)} \geq r
$$

is fulfilled, where $r$ denotes again a uniformly distributed point in the unit interval $[0,1]$. The number of trials in the acceptance-rejection method, i.e. the averaged number of points, which need to be generated in order to accept one point as $f-$ distributed, is exactly given by the constant $c$. This means, that the acceptancerejection method becomes quite time-consuming, if $c$ is large.

Applying an acceptance-rejection method the implementation of the CercignaniLampis model may be summarized as follows: in a first step, one generates for the given parameter $a$ and $b$ of the CL model a table of discrete values for the expression $H\left(\varepsilon, \varepsilon_{1}\right)$. Then, to perform binary collisions, one proceeds in the following way.

1) for each collision pair, one decides via the total cross-section, whether a collision will occur or not,

2) if two particles will collide, one evaluates the expression $H\left(\varepsilon, \varepsilon_{1}\right)$, where $\varepsilon$ and $\varepsilon_{1}$ denote the pre-collisional (scaled) rotational energies, using the table for $H$ and linear interpolation. With the value for $H\left(\varepsilon, \varepsilon_{1}\right)$ one decides whether the collision is performed according to the normalized density $\theta_{0} / H\left(\varepsilon, \varepsilon_{1}\right)$ or the second density $\theta_{2}(\ldots)$, i.e. if $r \leq H\left(\varepsilon, \varepsilon_{1}\right)$, where $r$ denotes a uniformly distributed random number in $[0,1]$, the collision is performed according to $\theta_{0} / H\left(\varepsilon, \varepsilon_{1}\right)$

$3)$ if the collision is according to the density $\theta_{0} / H\left(\varepsilon, \varepsilon_{1}\right)$, one applies an acceptance-rejection method with the density $\theta_{1}\left(\varepsilon^{\prime}, \varepsilon_{1}^{\prime}\right)$ on $0 \leq \varepsilon^{\prime} \leq 1,0 \leq \varepsilon_{1}^{\prime} \leq 1-\varepsilon^{\prime}$ given by

$$
\theta_{1}\left(\varepsilon^{\prime}, \varepsilon_{1}^{\prime}\right)=2
$$

i.e. the inversion method for the density defined in (2.6) yields the formula

$$
\varepsilon^{\prime}=\left(1-\sqrt{r_{1}}\right), \quad \varepsilon_{1}^{\prime}=\left(1-r_{2}\right)\left(1-\varepsilon^{\prime}\right)
$$

where $r_{1}$ and $r_{2}$ denote two uniformly distributed random numbers on $[0,1]$. The values $\varepsilon^{\prime}$ and $\varepsilon_{1}^{\prime}$ are accepted, if the condition

$$
\frac{1-\varepsilon^{\prime}-\varepsilon_{1}^{\prime}}{2-\varepsilon-\varepsilon_{1}-\varepsilon^{\prime}-\varepsilon_{1}^{\prime}} \exp \left[-a\left(\varepsilon-\varepsilon^{\prime}\right)^{2}-a\left(\varepsilon_{1}-\varepsilon_{1}^{\prime}\right)^{2}\right] \geq r
$$

is fulfilled, where $r$ denotes a uniformly distributed random number in $[0,1]$, otherwise one generates two new values for $\varepsilon^{\prime}$ and $\varepsilon_{1}^{\prime}$.

If two values $\varepsilon^{\prime}$ and $\varepsilon_{1}^{\prime}$ are accepted, the reduced total energy $E^{\prime}=E-\mathcal{E}^{\prime}-\mathcal{E}_{1}^{\prime}$, with $\mathcal{E}^{\prime}=\varepsilon^{\prime} E$ and $\mathcal{E}_{1}^{\prime}=\varepsilon_{1}^{\prime} E$, is distributed on the translational degrees of freedom of the two molecules using a standard collision step for a monoatomic gas, 
4) for the second term $\theta_{2}(\ldots)$, one applies an acceptance-rejection method with the standard density of the inelastic part of the Borgnakke-Larsen model given by $\theta_{b}\left(\varepsilon^{\prime}, \varepsilon_{1}^{\prime}\right)=6\left(1-\varepsilon^{\prime}-\varepsilon_{1}^{\prime}\right)$ on $0 \leq \varepsilon^{\prime} \leq 1,0 \leq \varepsilon_{1}^{\prime} \leq 1-\varepsilon^{\prime}$, and the condition to accept two points $\varepsilon^{\prime}$ and $\varepsilon_{1}^{\prime}$ is given by

$$
1-H\left(\varepsilon^{\prime}, \varepsilon_{1}^{\prime}\right) \geq r, \quad r \in[0,1]
$$

Here, the $a$-priori generation of a table for the expression $H\left(\varepsilon, \varepsilon_{1}\right)$ may be done using a (Quasi-)Monte Carlo integration of the integral given in (2.3), where the number of points to perform the numerical integration becomes quite large for $a \gg 1$.

\section{Numerical Simulations}

In the following we present some numerical simulations using the different crosssection models discussed in the previous sections. In particular, we will give results on the relaxation of the translational and rotational temperature in a homogeneous gas, like previously reported by Boyd [4] and Sack [14]. In a second set of simulations, we compute nitrogen shock waves for different Mach numbers and compare the results with experimental data given by Robben and Talbot [13] as well as some previous results obtained by Boyd [4] and Koura [8], [9]. The numerical experiments are performed on the basis of the Finite-Pointset method as discussed in [11].

\subsection{Relaxation in a Homogeneous Gas}

Here, we consider relaxation processes in a homogeneous gas consisting of nitrogen molecules, where at initial time the rotational temperature is set to zero, whereas the translational temperature is given by $5 / 3$ of the final equilibrium value. In particular, we consider the relaxation processes for different equilibrium values in a range from $300 \mathrm{~K}$ to $5.000 \mathrm{~K}$, with a total cross section given by the Sutherland Potential.

As mentioned above, a typical value for the parameter $a$ in the standard BorgnakkeLarsen model was given by Bird [2] for a nitrogen gas as $a=0.2$. On the other hand, in the generalized Borgnakke-Larsen model, the energy dependent parameter $a(E)$ is obtained from Parker's model for the rotational relaxation times. In the new models discussed in Section 2.2, we have two parameters $a$ and $b$, which may vary within certain ranges.

In Fig. 1 a) we compare the results obtained from the generalized Borgnakke-Larsen model with the original one and varies values for the inelastic collision parameter $a$ at an equilibrium temperature of $300 \mathrm{~K}$. One obtains an appropriate agreement between the two models for a constant parameter $a$ between 0.2 and 0.4 . If the equilibrium temperature is increased to $1000 \mathrm{~K}$ (Fig. 1 b)) and $5000 \mathrm{~K}$ (Fig. 1 c)), respectively, the relaxation process slows down and the appropriate value for $a$ 
should be chosen less then 0.2 for $T_{\infty}=1000 \mathrm{~K}$ and less then 0.1 for $T_{\infty}=5000 \mathrm{~K}$. The numerical results are obtained using 1000 particles and 50 independent samples to reduce the statistical fluctuations. Moreover, we performed 400 discrete time steps with a (scaled) time step of $\Delta t=0.05$.

This results clearly indicate, that one should use an energy-dependent inelastic collision parameter in order to ensure the correct rotational relaxation times.

In the next figures, we show some corresponding results obtained from the new crosssection models as discussed in Section 2.2, where we denote the model according to the approximate kernel (2.4) together with (2.2)-(2.3) as the N1 model, the one according to (2.5) together with (2.4) and (2.3) as the N2 model. In these models one may vary the two parameters $a$ and $b$.

Fig. 2 shows some results obtained for the N1 model: here, the coefficient $a$ turns out to be the sensitive parameter, whereas $b$ may be chosen as the maximal value to ensure that the term $H\left(\varepsilon, \varepsilon_{1}\right)$ remains less than 1. In order to fit the slow relaxation to equilibrium at high temperatures, the parameter $a$ has to be quite large, which indicates that a certain number of collisions are actually nearly elastic with respect to rotational energy exchange, because the expression $\exp \left(-a\left(\varepsilon-\varepsilon^{\prime}\right)^{2}-a\left(\varepsilon_{1}-\varepsilon_{1}^{\prime}\right)^{2}\right)$ is close to $\delta\left(\varepsilon-\varepsilon^{\prime}\right) \delta\left(\varepsilon_{1}-\varepsilon_{1}^{\prime}\right)$.

Using the N2 model, it is possible to fix the parameters $a$ and $b$ so that the relaxation curves nearly coincide with the one obtained with the (generalized) BorgnakkeLarsen model. In Fig. 3 we give the relaxation curves for the parameters $a=0.1$, $b=30$ at $T_{\infty}=300 \mathrm{~K}, a=1, b=3$ at $T_{\infty}=1000 \mathrm{~K}$ and $a=10, b=0.5$ at $T_{\infty}=$ $5000 \mathrm{~K}$, which should be compared with the results shown in Fig. 1.

\subsection{Simulations for Nitrogen Shock Waves}

The numerical simulations on relaxation processes in a homogeneous gas can not be verified experimentally. Hence, one should consider a different test case, for which experimental data are available. Robben and Talbot gave experimental data for the rotational temperature as well as the density for nitrogen in a shock wave [13], Alsmeyer reported some results on density profiles of shock waves in Ref. [1] and Boyd [4] and Koura [8], [9] used the experimental data to validate their numerical results obtained for nitrogen shock waves.

Here, we compare the different cross-sections considered in the paper for two different Mach numbers, namely, a weak shock at $M a=1.71$ and an upstream temperature of $T_{1}=200 \mathrm{~K}$ as well as a strong shock at $M a=12.9$ and a low temperature of $T_{1}=9 \mathrm{~K}$. Like in the previous section, we use the Sutherland potential as model for the total cross-section. The simulations are performed for a stationary shock

wave using scaled quantities, such that the downstream equilibrium values applying 
the Rankine-Hugoniot conditions are given by

$$
\begin{aligned}
\rho_{2} & =\frac{(\gamma+1) M a}{(\gamma-1) M a^{2}+2} \rho_{1} \\
u_{2} & =\frac{\rho_{1}}{\rho_{2}} u_{1} \\
T_{2} & =\left(2 \gamma M a^{2}-\gamma+1\right)\left((\gamma-1) M a^{2}+2\right) T_{1}
\end{aligned}
$$

where $\rho_{1}$ and $T_{1}$ denote the upstream density and temperature, respectively, the upstream velocity $u_{1}$ is given by $u_{1}=\sqrt{\gamma / 2} M a$ and $\gamma=7 / 5$ for a polyatomic gas. The resulting (scaled) downstream conditions are summarized together with the upstream conditions in Table 1.

Tab. 1 Rankine-Hugoniot Conditions

\begin{tabular}{c|ccc|ccc}
$M a$ & $\rho_{1}$ & $u_{1}$ & $T_{1}$ & $\rho_{2}$ & $u_{2}$ & $T_{2}$ \\
\hline 1.71 & 1 & 1.43 & 1 & 2.21 & 0.65 & 1.47 \\
12.9 & 1 & 10.79 & 1 & 5.82 & 1.85 & 33.30
\end{tabular}

The profiles shown in the following two subsections have been normalized as usual by

$$
\bar{\rho}=\frac{\rho-\rho_{1}}{\rho_{2}-\rho_{1}} \quad \bar{T}=\frac{T-T_{1}}{T_{2}-T_{1}}
$$

\subsection{1 $M a=1.71$ Shock Wave}

The simulations for the $M a=1.71$ shock wave with an upstream equilibrium temperature $T_{1}=200 \mathrm{~K}$ are performed on a spatial domain $[-10,10]$, using a scaled spatial coordinates based on the upstream mean free path for the hard-sphere potential; the shock wave is centered at $x=0$. The spatial domain is divided into 100 cells in order to perform the collisions among the gas. We use 400 particles per cell to approximate the upstream condition, which results in about 900 particles per cell for the corresponding downstream condition. The simulations are performed over 1000 discrete time steps and 10 independent samples, where the shock profiles are computed in each sample as an average over the last 200 time steps. The time step is chosen as the ratio of the length of a spatial cell and the upstream velocity $u_{1}=\sqrt{\gamma / 2}$ Ma.

Fig. 4 shows the various normalized shock profiles using the different differential cross-section models, where the $x$-coordinate is now scaled with respect to the upstream mean free path of the Sutherland potential, which is used as model for the total differential cross-section. Here, the mean free path ratio of the Sutherland and the hard-sphere potential at an equilibrium temperature of $200 \mathrm{~K}$ is given by $\lambda_{s p} / \lambda_{h s}=0.908$.

The results for the standard Borgnakke-Larsen model with $a=0.2$ and the generalized Borgnakke-Larsen model are quite similar to the one previously reported by 
Boyd [4] and Koura [8], [9]: there is a small temperature overshoot of about 2\%; the rotational temperature profile lags significantly the translational temperature profiles and slightly the density. The results for the N1 and N2 model ( $a=100$, $b=b_{\max }$ and $a=0.1, b=30$ ) do not clearly indicate an overshoot of the translational temperature. The numbers indicate, that there exists an overshoot of about $0.4 \%$, but one should increase the numerical accuracy to confirm this result. Moreover, the density and rotational temperature profiles nearly coincide; although the density seems to be slightly more steep.

A more detailed comparison on the different shock wave profiles is given for the density and rotational temperature in the upper half of Fig. 5. The N1 and N2 model yield identical results for both the density and the rotational temperature. The density profile applying the GBL model and the original Borgnakke-Larsen model are nearly identical; whereas the rotational temperature profile of the $\mathrm{BL}$ model slightly lags the one of the GBL model, which is in agreement with the results reported by Boyd [4]. The density profile of the N1/N2 model is more steep than in the GBL/BL model and deviations between the models are clearly observed in the rotational temperature.

\subsection{2 $M a=12.9$ Shock Wave}

In a second configuration we investigate a strong shock wave at an upstream equilibrium temperature of $T_{1}=9 \mathrm{~K}$. The simulations are performed on the same spatial domain as in the previous case; but, due to the low upstream temperature, the mean free path ratio between the Sutherland and the hard-sphere potential is now given by $\lambda_{s p} / \lambda_{h s}=0.109$. The spatial discretization is the same as in the previous simulations, but the number of particles used to approximate the upstream condition is reduced to 100 , which results in about 600 particle per cell for the downstream condition. Moreover, we perform only one single sample, because the statistical fluctuations are much lower then in the previous one - due to the high Mach number. Fig. 6 shows the shock wave profiles for the $M a=12.9$ case: all models yield an overshoot in the translational temperature profile: about $13 \%$ in the GBL model, $15 \%$ in the BL model, $10 \%$ in the N1 model and $12 \%$ in the N2 model. There is a clear separation between the single shock profiles: the translational temperature precedes the rotational one, which itself precedes the density. Moreover, this separation is slightly more large in the N1 model compared with the GBL model.

Like in the previous configuration, we give a more detailed comparison on the various models in the lower half of Fig. 5 for the density and rotational temperature profiles, respectively: one observes, that the density profiles nearly coincide for all models, whereas the rotational temperatures clearly differ. Now the results for the GBL and the N2 model nearly coincides, whereas the profile of the BL model precedes, the profile of the N1 model lags behind the profiles of the GBL and N2 model. 


\subsubsection{Comparison with Experimental Data}

Finally we compare our numerical results with the experimental data given by Robben and Talbot [13] and here we restrict ourselves to the generalized BorgnakkeLarsen and the N1 model. In Fig. 7 we have plotted the results for both models at the two Mach numbers $M a=1.71$ and $M a=12.9$, where the dots denote the corresponding experimental data.

The GBL model behaves quite similar to the models proposed by Koura in [8], [9]: at the lower Mach number we have a quite reasonable agreement with the experimental data except that the rotational temperature deviates close to the upstream region. At the high Mach number $M a=12.9$ we have an excellent agreement for the density profile, but in the experimental data the rotational temperature lags by a significant amount more behind the density profile than predicted by the numerical simulation.

With the N1 model we have a better agreement between the experimental data and the numerical simulation for both Mach numbers: at $M a=1.71$ the agreement in the rotational temperature close to the upstream condition is much better than compared with the GBL model, at $M a=12.9$ even the rotational temperature profiles nearly coincide in our new model. This is a quite interesting result which one may not expect from the previous results on the homogeneous relaxation.

\section{Conclusion}

In the present paper we discussed various differential cross-section models for a classical polyatomic gas. Besides the well-known Borhnakke-Larsen model we considered a new approach recently proposed by the same authors which is not a-priori based on a separation of rotational-elastic and completely inelastic collisison like assumed in Borgnakke-Larsen-type models.

We explained how to implement the various models in a simulation code like the DSMC method of Bird. The comparisons between the different models are performed for a simple homogeneous relaxation as well as the standard shock wave problem in rarefied gases.

For the latter one, we further compared the numerical results with existing experimental data. In particular this comparison turned out to give quite interesting results, because we were able to obtain a better agreement for the new model then given by Borgnakke-Larsen-type models.

\section{Acknowledgement}

The research of C.C. and M.L. was supported by a grant of the Italian National Council of Research (C.N.R.). 


\section{References}

[1] H. Alsmeyer, "Density profiles in argon and nitrogen shock waves measured by the absorption of an electron beam", J. Fluid Mech., 74, 497-513 (1976).

[2] G.A. Bird, Molecular Gas Dynamics and the Direct Simulation of Gas Flows, Oxford University Press, Oxford (1994).

[3] C. Borgnakke and P.S. Larsen, "Statistical Collision Model for Monte Carlo Simulation of Polyatomic Gas Mixtures", J. Comp. Phys, 18, 405-420 (1975).

[4] I.D. Boyd, "Rotational-Translational Energy Transfer in Rarefied Nonequilibrium Flows", Phys. Fluids A, 2, 447-452 (1990).

[5] C. Cercignani, Theory and Application of the Boltzmann Equation, Scottish Academic Press, Edinburgh (1975).

[6] C. Cercignani and M. Lampis, "A New Model for the Differential Cross Section of a Polyatomic Gas", Rarefied Gas Dynamics, Peking University Press, Beijing, 731-736 (1997).

[7] C. Cercignani, M. Lampis and J. Struckmeier, "New models for the differential cross section of a polyatomic gas in the frame of the scattering kernel theory", Mechanics Research Communications, 25, 231-236 (1998).

[8] K. Koura, "Statistical Inelastic Cross-Section for the Monte Carlo Simulation of Molecules with Discrete Internal Energy", Phys. Fluids A, 4, 1782-1788 (1992).

[9] K. Koura, "Statistical Inelastic Cross-Section for the Monte Carlo Simulation of Molecules with Continuous Internal Energy", Phys. Fluids A, 5, 778-780 (1993).

[10] I. Kuščer, "Dissociation and Recombination in an Inhomogeneous Gas", Physica $A, 542-556$ (1991).

[11] H. Neunzert and J. Struckmeier, "Particle Methods for the Boltzmann Equation", ACTA NUMERICA 1995, Cambridge, 1995.

[12] J.G. Parker, "Rotational and Vibrational Relaxation in Diatomic Gases", Phys. Fluids, 2, 449 (1959).

[13] F. Robben and L. Talbot, "Experimental Study of the Rotational Distribution Function of Nitrogen in a Shock Wave", Phys. Fluids, 9, 653-662 (1966).

[14] W. Sack, Modeling and Numerical Simulation of Reactive Flows in Rarefied Gases (in German) PhD-thesis, Dept. Math., Univ. Kaiserslautern, 1995. 

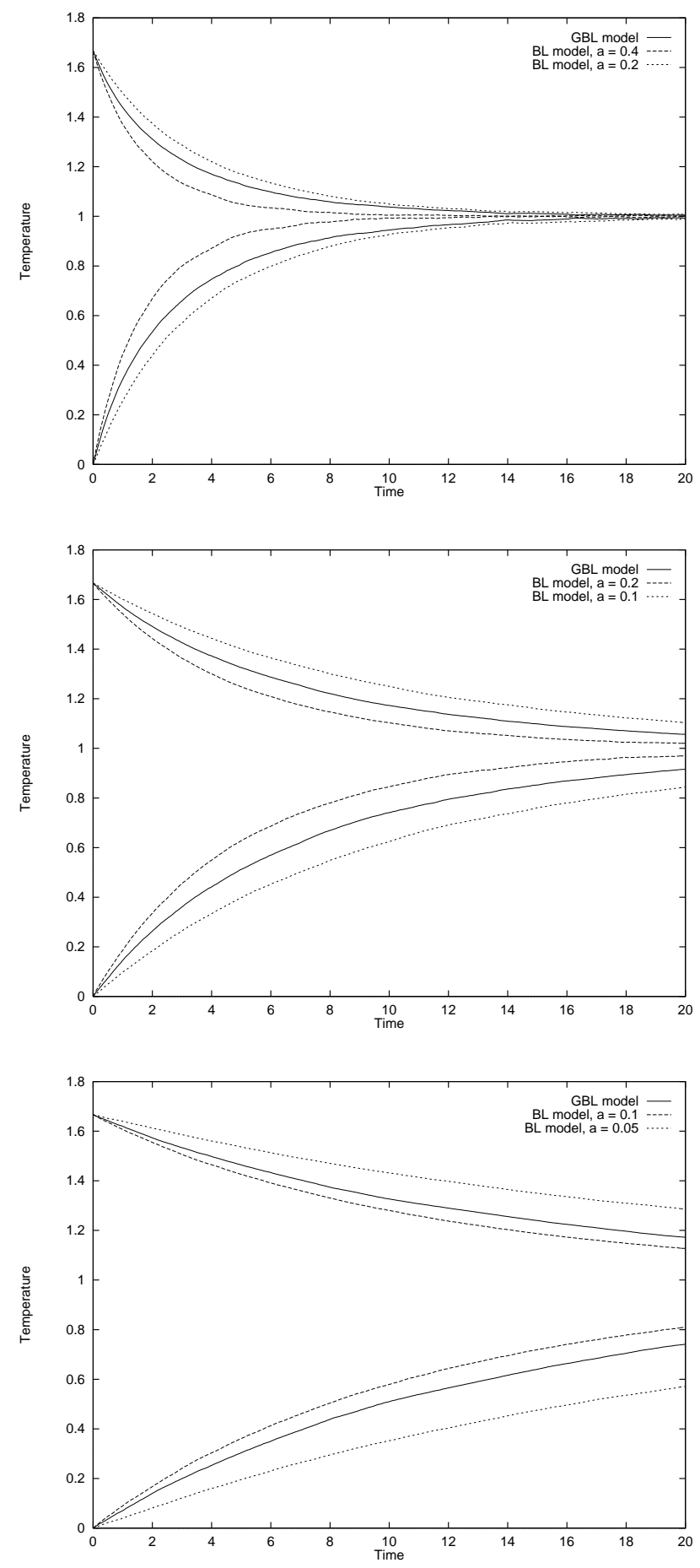

FIG. 1. Homogeneous Relaxation for the GBL and BL model at $T_{\infty}=300 \mathrm{~K}$, $T_{\infty}=1000 \mathrm{~K}$ and $T_{\infty}=5000 \mathrm{~K}$. 


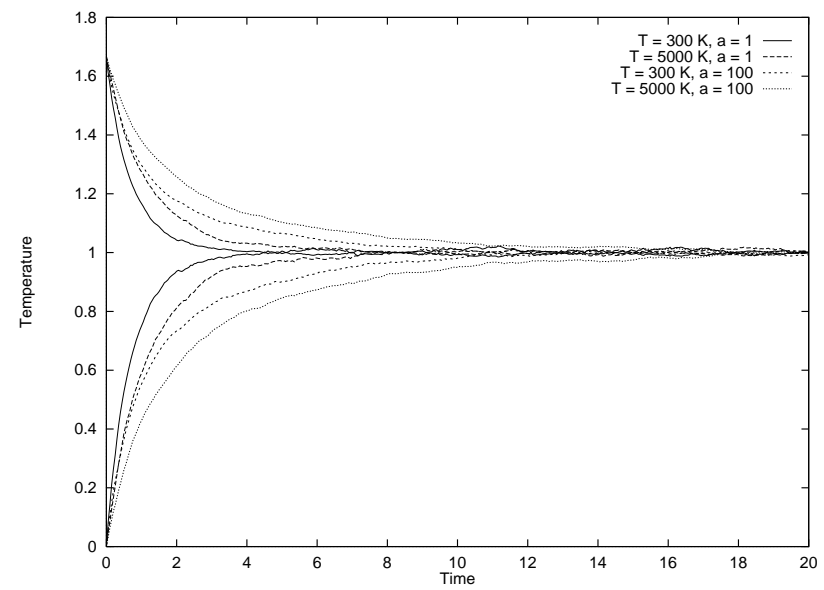

FIG. 2. Homogeneous Relaxation for the N1 model for different equilibrium temperatures and different parameters $a$ and $b$.

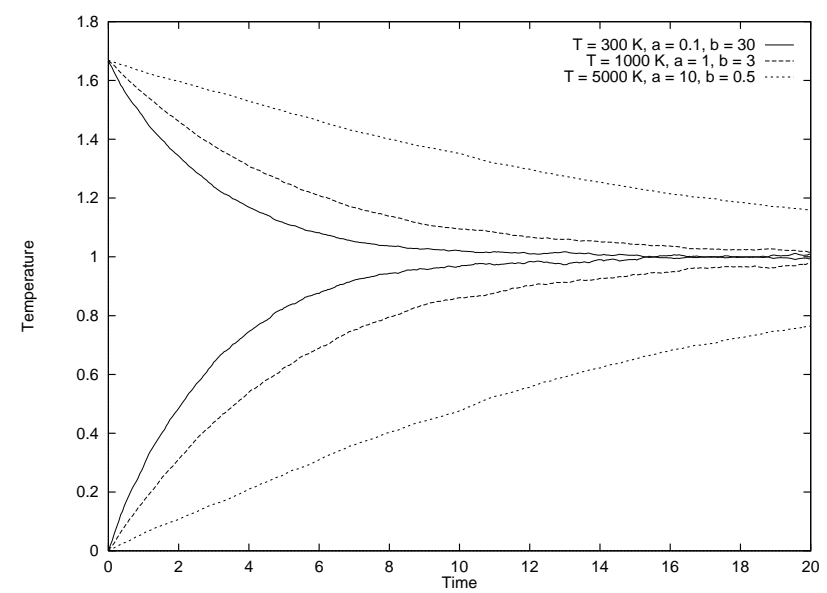

FIG. 3. Homogeneous Relaxation for the N2 model for different equilibrium temperatures and different parameters $a$ and $b$. 

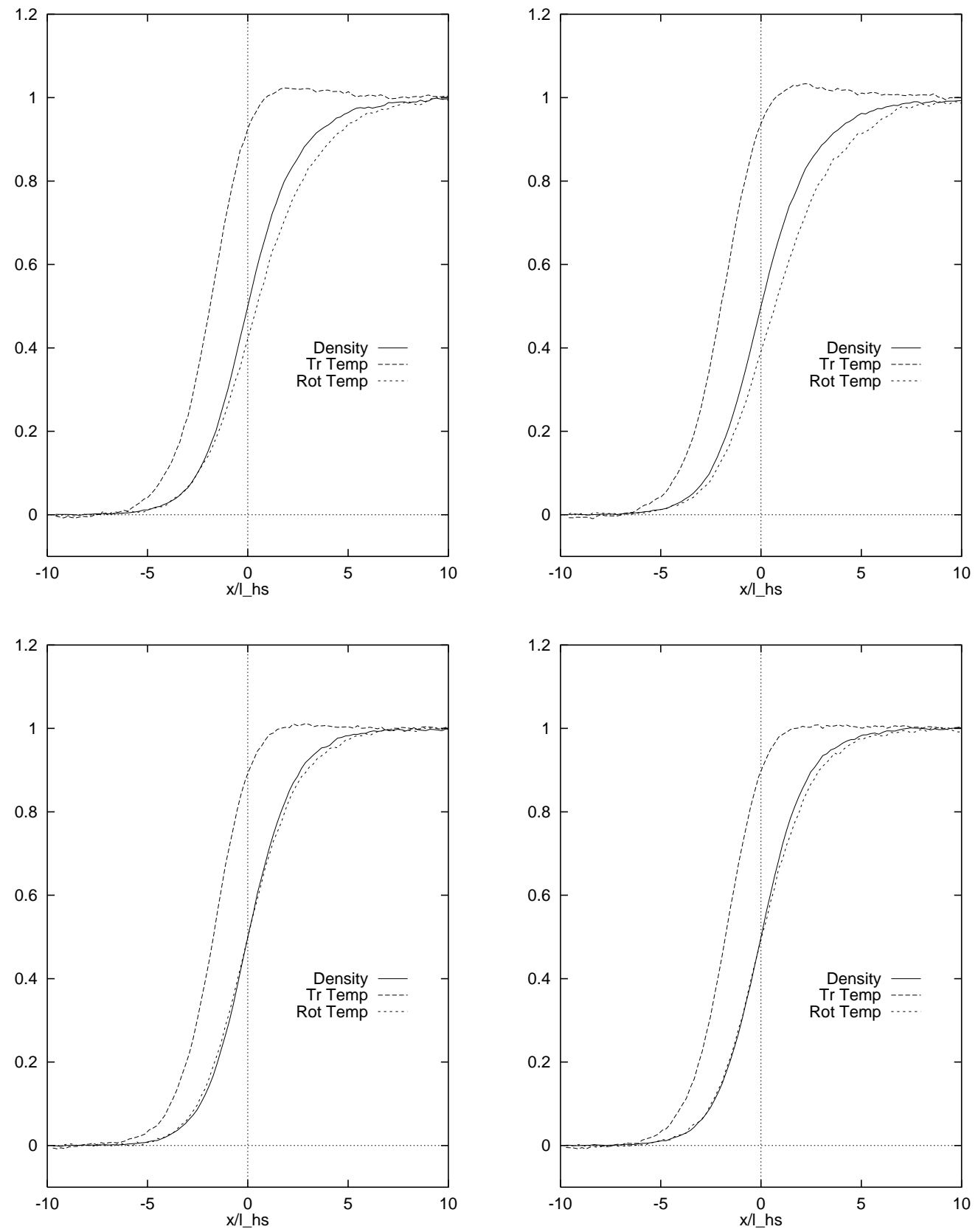

FIG. 4. Shock wave profiles for different cross-section models at $M a=1.71$ :

GBL model (upper left), BL model (upper right), N1 model (lower left), N2 model (lower right) 

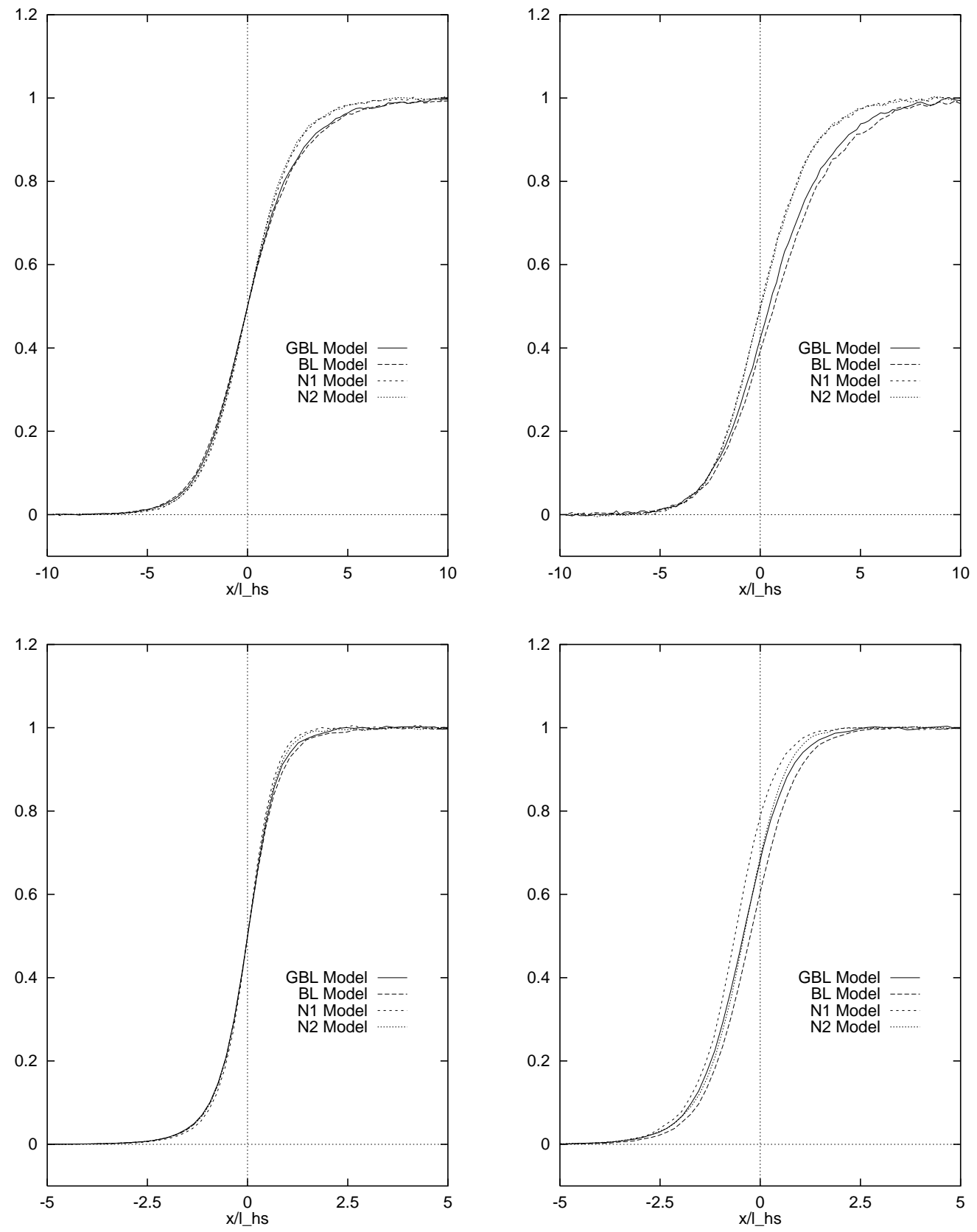

FIG. 5. Comparison of shock profiles for different cross-section models:

density profiles at $M a=1.71$ (upper left), rotational temperature profiles at $M a=1.71$ (upper right), density profiles at $M a=12.9$ (lower left), rotational temperature profiles at $M a=12.9$ (lower right) 

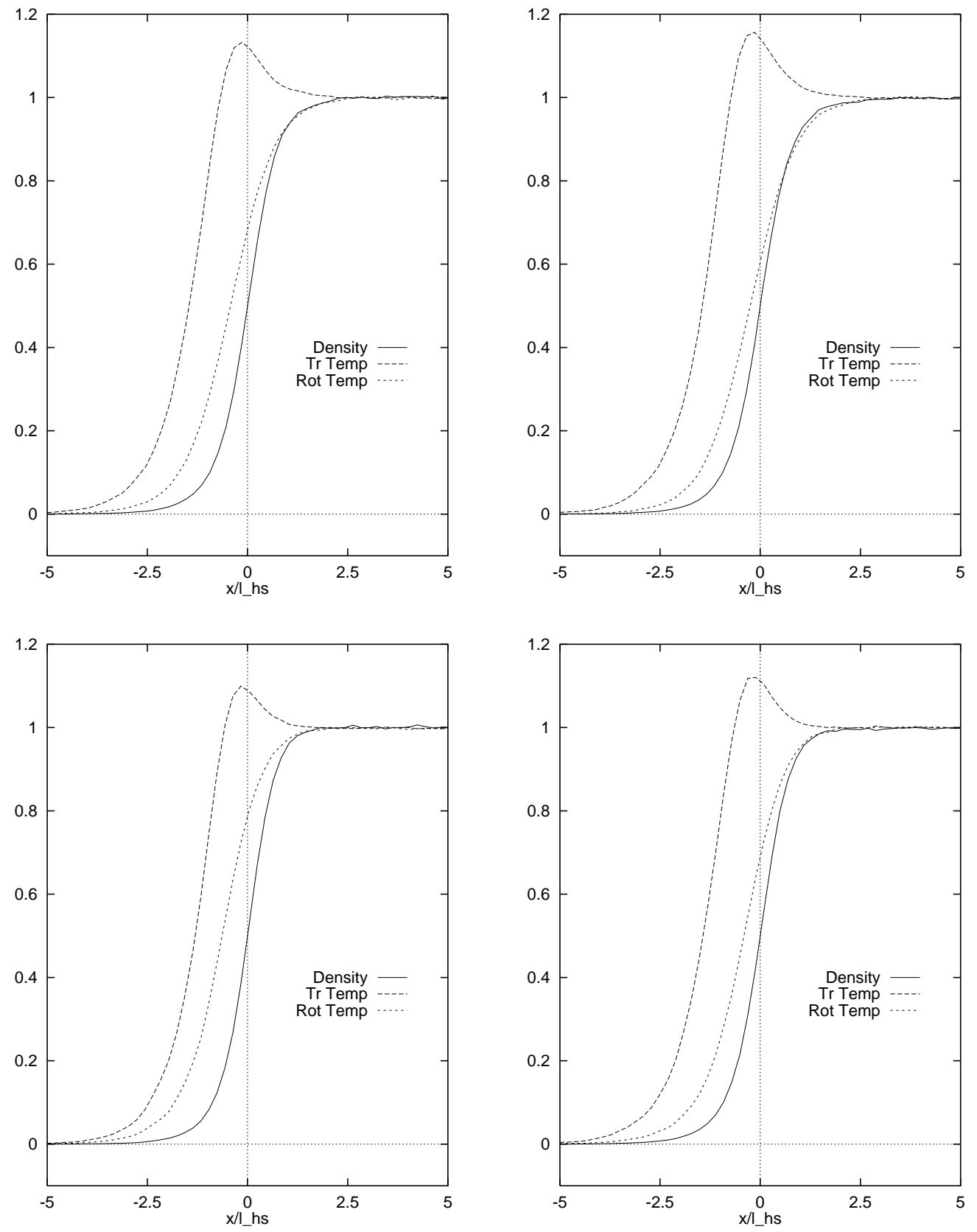

FIG. 6. Shock wave profiles for different cross-section models at $M a=12.9$ :

GBL model (upper left), BL model (upper right), N1 model (lower left), N2 model (lower right) 

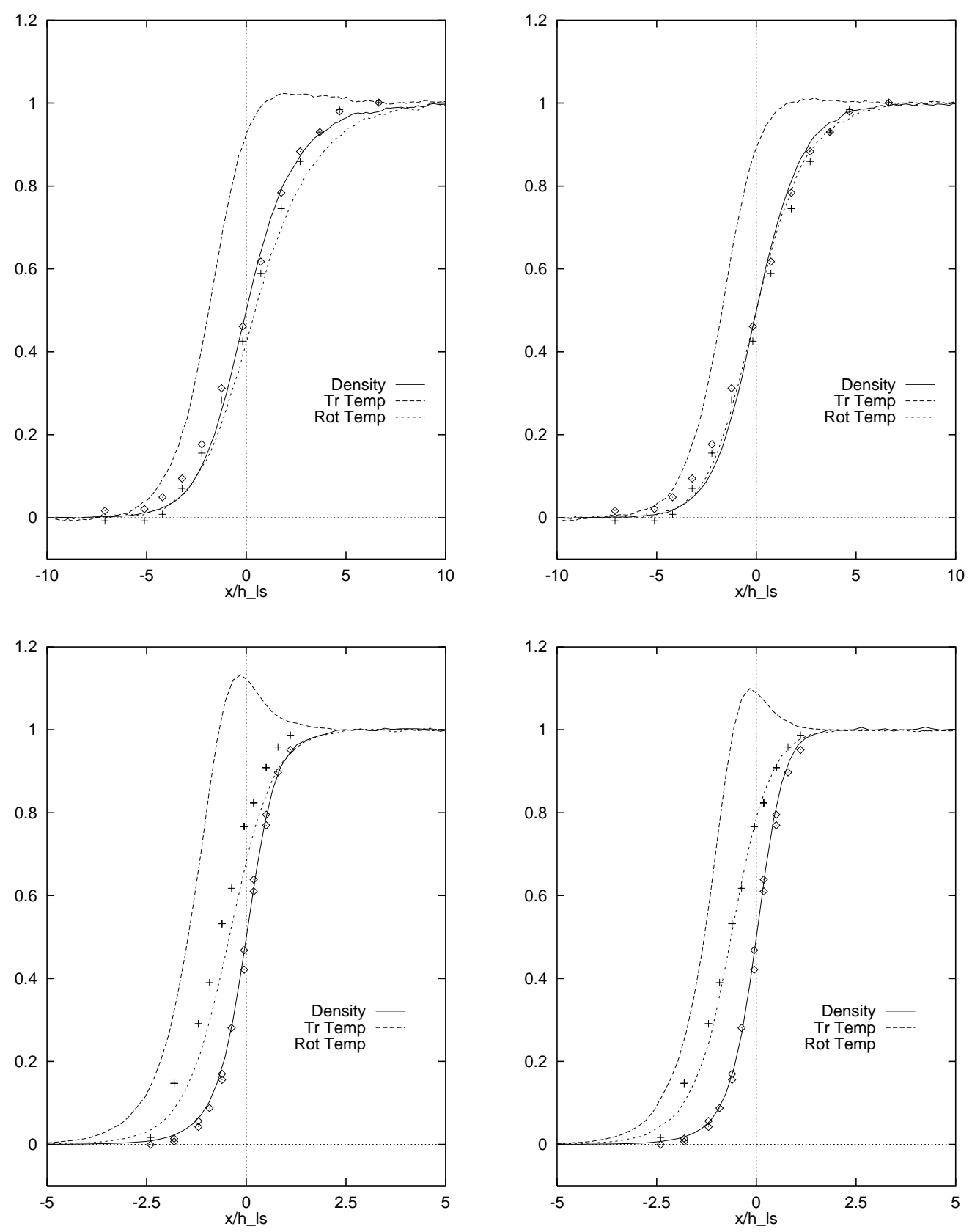

FIG. 7. Shock wave profiles for the GBL model and the N1 model compared to experimental data: GBL model at $M a=1.71$ (upper left), N1 model at $M a=1.71$ (upper right), GBL model at $M a=12.9$ (lower left) and N1 model at $M a=12.9$ (lower right), $(\diamond)$ experimental data for the density, $(+)$ experimental data for the rotational temperature 